\title{
Clinical significance of mean platelet volume/lymphocyte ratio and mean platelet volume/platelet ratio in the exacerbation of chronic obstructive pulmonary disease
}

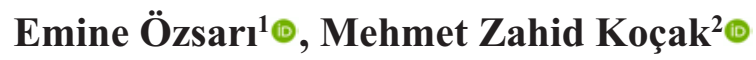 \\ ${ }^{1}$ Department of Chest Diseases, Bolu Abant İzet Baysal University School of Medicine, Bolu, Turkey \\ ${ }^{2}$ Department of Internal Medicine, Bolu Abant İzet Baysal University School of Medicine, Bolu, Turkey
}

DOI: $10.18621 /$ eurj.443660

\begin{abstract}
Objectives: Studies showing the role of systemic inflammation in chronic obstructive pulmonary disease (COPD) are increasing. Particularly, importance of mean platelet volume (MPV) and neutrophil/lymphocyte ratio (NLR) for acute exacerbation of COPD has been reported. The use of MPV/lymphocyte ratio (MLR) and $\mathrm{MPV} /$ platelet ratio(MPR) in acute exacerbation of COPD patients was investigated in our study, considering that MPV alone may be a more valuable marker of inflammation.

Methods: Between March 2017 and March 2018, COPD patients who applied to Abant Izzet Baysal University School of Medicine, Chest Diseases outpatient clinic were examined. Results were retrospectively scanned from patient files after institutional approval. Sixty-four (60.4\%) stable COPD and $42(39.6 \%)$ acute exacerbation COPD patients were included in the study.

Results: Seventy-one (67\%) of patients were male and $35(33 \%)$ were female. NLR was $2.26(0.93-6.48)$ in stable patients and $4(1.18-36)$ in acute attack patients $(p<0.001)$; PLR was $137.44(66.9-436.6)$ in patients with stable disease and 162.8 (85-1056.6) in patients with attack $(p=0.068)$. MLR was $5(2.92-25)$ in acute attack patients and $4(1.89-8.67)$ in stable patients; this difference was statistically significant $(p=0.003)$. MPV was found to be 7 (5.5-9.1) fL in patients with stable disease and 8 (5-13.4) fL in acute patients. This difference was statistically significant $(p<0.001)$. MPR was found to be statistically significantly higher in acute patients than in patients with stable disease $(p=0.04)$. WBC, neutrophil and CRP were found to be statistically significantly higher in acute patients than in stable patients $(p<0.05)$. There was a statistically significant correlation between WBC and NLR $(\mathrm{r}=0.269, p=0.005)$ and between CRP and NLR $(\mathrm{r}=0.379, p<0.001)$. Conclusions: Hemogram parameters from routine laboratory tests in COPD patients are cheap and easily accessible. It is important to detect the presence of subclinical inflammation in the stable phase, as well as to identify patients at risk of exacerbation. Prospective studies are needed to demonstrate correlations with inflammatory markers.
\end{abstract}

Keywords: Mean platelet volume, neutrophil/lymphocyte ratio, lymphocyte, platelet, chronic obstructive pulmonary disease

Received: July 13, 2018; Accepted: January 29, 2019; Published Online: July 24, 2019

Address for correspondence: Emine Özsarl, MD, Bolu Abant İzzet Baysal University School of Medicine, Department of Chest Diseases, Bolu, Turkey E-mail:dreminedemirok@hotmail.com 
O hronic obstructive pulmonary disease (COPD), reveals by developing a variety of particles as a result of exposure to persistent airflow limitation. It is thought that both the prevalence, the economic and social burden of the world will increase year by year [1]. One of the causes that affect the development of the disease is exacerbations. Early detection of this is a preventive measure in terms of major complications [2]. Recently, the potential role of hemogram parameters during follow-up of chronic diseases has been investigated. The mean platelet volume (MPV) and neutrophil/lymphocyte ratio (NLR) especially have been reported for acute exacerbation of COPD [3, 4]. It has been reported that thrombocytopenia is associated with poor outcomes in acute exacerbations [5], while thrombocytosis is associated with short and long term mortality after exacerbations [6]. The number of lymphocytes is known to decrease in inflammatory conditions [7]. In our study, the MPV/lymphocyte ratio (MLR) and MPV/platelet ratio (MPR) alone were considered to be a more valuable marker of inflammation than MPV. The purpose of hypothesis is; MLR and MPR could be used as biomarkers in acute exacerbation in COPD patients.

\section{METHODS}

COPD patients who applied to the Abant Izzet Baysal University School of Medicine Chest Diseases outpatient clinic between March 2017 and March 2018 were included in the study. Results were retrospectively scanned from patient files after institutional approval. Patients with stable COPD were evaluated according to the latest updated COPD combined staging [1]. Exacerbation of COPD was determined according to treatment options based on the detailed screening of symptoms such as dyspnea, cough, or sputum production and / or purulence, and whether acute deterioration resulted in symptoms requiring additional treatment [8] According to this; patients with exacerbation were grouped as mild, moderate and severe. Demographic characteristics, comorbidities, hemogram parameters and C-reactive protein (CRP) values were recorded. Blood was collected before the patients began taking systemic corticosteroids (intravenous), antibiotics (oral/intravenous), and theophylline. MLR was performed by lymphocytic cleavage of MPV; NLR, by neutrophilin lymphocyte cleavage; PLR was obtained by platelet lymphocytic cleavage and MPR by platelet cleavage of MPV. Patients who had another exacerbation during 3 months, who had obstructive sleep apnea, cor pulmonale, pneumonia, pulmonary thromboembolism, coagulopathy, malignancy, and drug use that could affect mean platelet volume were excluded from the study. The study was approved by the Chief Medical Officer of Abant İzzet Baysal University Medical Faculty Education and Research Hospital. The research protocol is in line with the 2000 Helsinki Declaration.

\section{Statistical Analysis}

In statistical analysis, statistical package program of IBM Statistics 16.0 (SPSS) was used. Normal distribution of continuous variables was evaluated by Kolmogorov Smirnov tests. Indepented t test was used for normal variables and Mann-Whitney $U$ test was used for normal variables. For comparison of multiple groups, Kruskal-Wallis test was used for normal nonscattering data. The Spearman correlation test was used for the relationship between non-normally distributed continuous variables. Statistical significance was accepted as $p<0.05$.

\section{RESULTS}

Sixty-four (60.4\%) stable COPD and 42 (39.6\%) acute exacerbation COPD patients were included in the study. Seventy-one $(67 \%)$ of the patients were male and $35(33 \%)$ were female. While $71(67 \%)$ patients had additional disease, 35 (33\%) had no additional disease. $12(11.3 \%)$ of the patients never smoked, 65 (61.3\%) were ex-smoker, 29 (27.4\%) were active smokers. Patients with acute exacerbation had 4 (9.5\%) heavy, 21 (50\%) mild, 17 (40.5\%) moderate activation. Twenty-two (34\%) of the stable patients were stage A, $22(34 \%)$ were grade B, 17 (27\%) were stage $\mathrm{C}$ and $3(5 \%)$ according to GOLD. The mean age of acute exacerbation patientspatients was $66.5 \pm 9.3$ years, while the mean age of stabilized patients was $63.9 \pm 10.2$ years $(p=0.19)$. There was no smoking history in 5,23 were ex-smoker, 14 were active smokers. NLR was $2.26(0.93-6.48)$ in stable patients and $4(1.18-36)$ in acute exacerbation $(p<0.001)$. PLR 
Table 1. Laboratoryfindings of acuteexacerbationandstabilized COPD patients

\begin{tabular}{lccc}
\hline & Stabil COPD & Active COPD & $\boldsymbol{p}$ value \\
\hline Age (years) & $63.9 \pm 10.2$ & $66.5 \pm 9.3$ & 0.19 \\
Platelet (K/uL) & $274.3 \pm 69.8$ & $277.05 \pm 71.1$ & 0.84 \\
White blood cell (K/uL) & $7.1 \pm 1.38$ & $11.6 \pm 4.1$ & $<\mathbf{0 . 0 0 1}$ \\
Neutrophil/lymphocyte ratiio & $2.26(0.93-6.48)$ & $4(1.18-36.6)$ & $<\mathbf{0 . 0 0 1}$ \\
Platelet/lymphocyte ratio & $137.4(66.9-436.6)$ & $162.8(85-1056.6)$ & 0.068 \\
MPV/lymphocyte ratio & $4(2.89-8.67)$ & $5(2.92-25)$ & $\mathbf{0 . 0 0 3}$ \\
MPV/platelet ratio & $0.026(0.014-0.041)$ & $0.028(0.020-0.067)$ & $\mathbf{0 . 0 4}$ \\
Neutrophil (K/uL) & $4(2.1-9.7)$ & $6(2-14.5)$ & $<\mathbf{0 . 0 0 1}$ \\
MPV (fL) & $7(5.5-9.1)$ & $8(5.813 .4)$ & $<\mathbf{0 . 0 0 1}$ \\
C-reactive protein $(\mathrm{mg} / \mathrm{L})$ & $3.3(0.1-20)$ & $26(6.3-267.7)$ & $<\mathbf{0 . 0 0 1}$ \\
\hline Shown
\end{tabular}

Shown as mean \pm standard deviation or median (minimum-maximum). COPD $=$ Chronic obstructive pulmonary disease, MPV $=$ Mean platelet volume

was $137.44(66.9-436.6)$ in patients with stable disease and 162.8 (85-1056.6) in patients with exacerbation $(p=0.068)$. MLR was $5(2.92-25)$ in patients with acute attack and 4 (1.89-8.67) in patients with stable disease, and this difference was statistically significant $(p=0.003)$. Similarly, MPR was statistically significantly higher in acute patients than in stable patients $(p=0.04)$ (Table 1). MPV was found to be 7 (5.5-9.1) fL in patients with stable disease and 8 (513.4) $\mathrm{fL}$ in acute patients. This difference was statistically significant $(p<0.001)$. Among the other inflammatory parameters, WBC, neutrophil and CRP were statistically significant $(p<0.05$ for all) in acute patients compared to the stable patients (Table 1).

Correlation between WBC, CRP, NLR, MLR and MPR was statistically significant between WBC and NLR a correlation was found $(\mathrm{r}=0.269, p=0.005)$. There was a statistically significant correlation between CRP and NLR ( $\mathrm{r}=0.379, p<0.001)$. Similarly, there was a statistically significant correlation between NLR and MLR $(\mathrm{r}=0.805, p<$ $0.001)$. There was no correlation between MPR and inflammatory parameters. In patients with acute exacerbation, NLR, MLR and MPR were assessed

Table 2. Correlation of inflammatory parameters

\begin{tabular}{llcccc}
\hline & & Number & Mean & Standard. deviation & $p$ value \\
\hline \multirow{2}{*}{ NLR } & Mild & 21 & 3.7538 & 2.43081 & 0.095 \\
& Modarate & 17 & 6.4442 & 8.00233 & \\
\multirow{3}{*}{ MLR } & Severe & 4 & 6.8714 & 3.84920 & \\
& Mild & 21 & 5.7136 & 3.35507 & 0.178 \\
& Modarate & 17 & 7.5003 & 5.26600 & \\
\multirow{3}{*}{ MPR } & Severe & 4 & 4.7548 & 1.96772 & 0.528 \\
& Mild & 21 & 0.02991 & 0.008899 & \\
& Modarate & 17 & 0.03443 & 0.012472 & \\
\hline
\end{tabular}

$\mathrm{NLR}=$ Neutrophil/lymphocyte ratio, $\mathrm{MPV}=$ Mean platelet volume, $\mathrm{MLR}=\mathrm{MPV} /$ lymphocyte ratio, $\mathrm{MPR}=$ $\mathrm{MPV} /$ platelet ratio 
separately for disease severity. NLR levels were found to be higher in moderate and mild patients in severely ill patients but not statistically significant. MLR and MPR levels were lower in mild cases than in moderate to severe cases and moderate to severe cases. These values were not statistically significant $(p>0.05$ for all) (Table 2).

\section{DISCUSSION}

In recent years, studies showing the role of systemic inflammation in COPD are increasing. Proinflammatory cytokines such as CRP, fibrinogen and IL-6 have been shown to increase in systemic circulation in patients with stable COPD [9, 10]. Thrombocytes are activated by the release of proinflammatory cytokines, especially C-reactive protein [CRP], TNF- $\alpha$ and IL-6 [11]. MPV is one of the important predictors of platelet function. Studies have reported that the MPV and platelet counts are inversely related to the normal population $[12,13]$, so MPV and platelet counts need to be interpreted as a significant proportion of independent variables $[14,15]$. When the literature is reviewed, no study has been found to demonstrate that MPV/lymphocyte ratio (MLR) and MPV/platelet ratio (MPR) can be assessed in acute COPD exacerbation.

The views on the possibility that MPV can be used as an inflammatory marker are contradictory $[11,14-$ 18]; Gasparyan et al. [11] reported that MPV decreased as the degree of inflammation increased. Conversely, another study comparing COPD patients with healthy controls [14] showed a significant increase in MPV. In another study, it was stated that MPV decreased during the attack period and increased during the stabilization period [15]. Agapakis et al. [16] also found that lower MPVs were found in the stabilization and exacerbation groups, while Wang et al. [17] have found that COPD patients have a lower MPV in the stable and acute exacerbation phases.

According to inflammation in COPD, platelets may be expected to increase in number and volume as a positive acute phase reactant, considering that COPD is present during both stabilization and exacerbation periods. However, there are contradictory mechanisms explaining this condition. Because of the inflammation secondary to hypoxia ,the increase in prothrombotic activity caused by endothelial injury and the stimulatory effect of proinflammatory cytokines such as fibrinogen on platelets, can be explained the increase in the production of larger reactive platelets, when cytokines are considered to stimulate thrombocytes. MPV is elevated in low-grade chronic inflammatory diseases predominantly, thrombosis such as atherosclerosis [18]. MPV is reduced in highgrade inflammatory diseases such as active rheumatoid arthritis, inflammatory bowel disease and familial mediterranean fever. Intensive consumption of larger platelets in inflammatory regions may account for reduced MPV in patients with COPD. This study found that MPV was significantly increased in patients with acut exacerbation, and this increase was significant $(p<0.001)$. PLT values were found to be insignificant although there was an increase in these patients $(p=0.84)$.

Recently, platelet/lymphocyte ratio (PLR) has been evaluated as a prognostic marker in various inflammatory and cardiovascular diseases. Kurtipek et al. [20] reported that PLR was elevated in patients with active COPD compared with patients with stable COPD. In a study by Karadeniz et al. [20], PLR was shown not only in acute exacerbation patients but also in patients with stable COPD. In their study, PLR values were negatively correlated with severity of airway obstruction [21]. This increase was not statistically significant in spite of the fact that PLR value was significantly higher in patients with acute exacerbation ( $p=0.068)$.

It has been reported that lymphocyte counts can also be used to show inflammation in chronic diseases such as platelets and neutrophils [22]. It is predicted that MLR may be more valuable than these parameters in reflecting inflammation given the increased MPV and lymphocyte count alone. One study showed that MLR levels were a prognostic factor in diabetic and acute myocardial infarction (MI), patients with increased MLR had a greater thrombotic volume and worse angiographic characteristics; high MLR is reported to be a risk factor for early and late mortality after MI [13]. In our study, MLR levels in acute exacerbated COPD patients were significantly higher than those in stable COPD patients $(p=0.003)$. To our knowledge, it is the first study to show high MLR levels in acute exacerbation in COPD patients $(p=$ 0.178). We showed that MLR and MPR were higher 
in patients with acute exacerbation in COPD than in patients with stable disease, and these differences were statistically significant. ( $p=0.003$ for MLR, $p=0.04$ for MPR). Our study showed high MPR levels in patients with acute exacerbatif COPD. As in MLR, MPR does not show the severity of the disease with exacerbation in acute COPD $(p=0.528)$.

Assessment of the neutrophil/lymphocyte ratio (NLR) assessment in patients presenting with COPD exacerbation may provide important keys [23]. Lymphocytopenia is observed with increased WBC and neutrophil, especially in inflammation caused by bacterial infections. NLR has been reported to show active inflammation in cardiovascular diseases, renal diseases, familial Mediterranean fever, active ulcerative colitis and acute coronary syndrome [24, 25]. In one study, a significant increase in NLR was shown in stable COPD patients compared to healthy controls [26]. It is showed that a lower number of eosinophils and a higher NLR $(>7)$ were independent risk factors for sepsis mortality in the study of Terradas et al. [27]. Göçmen et al.'s study [28] that investigating the relationship between COPD and NLR severity, they reported that NLR correlated positively with arterial $\mathrm{PaCO} 2$ and negative correlation with $\mathrm{pH}$ and FEV1/FVC. In the study of Gao et al. [29], It was shown that NLR is high in both exacerbation and stabilization period, and there is a positive correlation between exacerbation severity and NLR. In our study, NLR levels were higher in acute exacerbativ COPD patients compared to stable COPD, it was also meaningful. $(p<0.001)$ NLR did not show the severity of acute exacerbated COPD, such as MLR and MPR $(p=0.095)$.

CRP is the most well-known inflammatory biomarker and is increasing in most cases of infection, inflammation and tissue damage. Correlation between WBC, CRP NLR, MPR and MLR supports the hypothesis that these new markers (MPR and MLR) can be used as a marker of acute exacerbation in COPD.

\section{Limitations}

Retrospective design is the most important limitation of this study. However, since our study is performed in a limited chest disease clinic, our results can not be generalized for all patients. The limited number of patients, especially if the number of patients with severe exacerbation is low. Finally there are complex relationships between comorbid diseases (diabetes mellitus, hypertension, hypercholesterolemia, obesity, metabolic syndrome, atrial fibrillation), medications (statins, some antihypertensives, antiplatelet drugs) and smoking in COPD. There is a need for comparative studies with matched controls for age and previous exposure to cigarette smoke, as these factors can greatly affect MPV values.

\section{CONCLUSION}

Exacerbation is a serious problem in COPD, this exacerbation is detected early and mortality and morbidity decrease if treatment is started. Hemogram parameters obtained as part of a routine laboratory test in patients with COPD are simple, inexpensive and easily accessible tools. Before the adverse clinical outcomes occur, they can be used either individually or by measuring the proportions to each other. It is predicted that there may be subclinical inflammation in the stable period, as well as useful indications to evaluate the severity of acute attacks and the management of acute episodes. They should also be able to detect patients who are at risk of exacerbation. Prospective studies are needed to demonstrate correlations between other inflammatory markers.

\section{Conflict of interest}

The authors disclosed no conflict of interest during the preparation or publication of this manuscript.

\section{Financing}

The authors disclosed that they did not receive any grant during conduction or writing of this study.

\section{REFERENCES}

[1] Global Strategy for the Diagnosis, Management and Prevention of COPD, Global Initiative for Chronic Obstructive Lung Disease (GOLD) 2017. Available from: http://goldcopd.org [2] Suissa S, Dell'Aniello S, Ernst P. Long-term natural history of chronic obstructive pulmonary disease: severe exacerbations and mortality. Thorax 2012;67:957-63.

[3] Yousef AM, Alkhiary W. Role of neutrophil to lymphocyte ratio in prediction of acute exacerbation of chronic obstructive 
pulmonary disease. Egyptian J Chest Dis Tuberc 2017;66:43-8. [4] Steiropoulos P, Papanas N, Nena E, Xanthoudaki M, Goula $\mathrm{T}$, Froudarakis $\mathrm{M}$, et al. Mean platelet volume and platelet distribution width in patients with chronic obstructive pulmonary disease: the role of comorbidities. Angiology 2013;64:535-9.

[5] Rahimi-Rad MH, Soltani S, Rabieepour M, Rahimirad S. Thrombocytopenia as a marker of outcome in patients with acute exacerbation of chronic obstructive pulmonary disease. Pneumonol Alergol Pol 2015;83:348-51.

[6] Paliogiannis P, Fois AG, Sotgia S, Mangoni AA, Zinellu E, Pirina $\mathrm{P}$, et al. Neutrophil to lymphocyte ratio and clinical outcomes in COPD: recent evidence and future perspectives. Eur Respir Rev 2018;27(147). pii:170113.

[7] Unsinger J, Kazama H, McDonough JS, Hotchkiss RS, Ferguson TA. Differential lymphopenia-induced homeostatic proliferation for CD4+ and CD8+ T cells following septic injury. J Leukoc Biol 2009;85:382-90.

[8] Anthonisen NR, Manfreda J, Warren CP, Hershfield ES, Harding GK, Nelson NA. Antibiotic therapy in exacerbations of chronic obstructive pulmonary disease. Annals of Internal Medicine 1987;106:196-204.

[9] Schols AM, Buurman WA, Staal van den Brekel AJ, Dentener MA, Wouters EF. Evidence for a relation between metabolic derangements and increased levels of inflammatory mediators in a subgroup of patients with chronic obstructive pulmonary disease. Thorax 1996;51:819-24.

[10] Donaldson GC, Seemungal TA, Patel IS, Bhowmik A, Wilkinson TM, Hurst JR, et al. Airway and systemic inflammation and decline in lung function in patients with COPD. Chest 2005;128:1995-2004.

[11] Gasparyan AY, Ayvazyan L, Mikhailidis DP, Kitas GD. Mean platelet volume: a link between thrombosis and inflammation? Curr Pharm Design 2011;17:47-58.

[12] Cho H, Hur HW, Kim SW, Kim SH, Kim JH, Kim YT, Lee $\mathrm{K}$. Pre-treatment neutrophil to lymphocyte ratio is elevated in epithelial ovarian cancer and predicts survival after treatment. Cancer Immunol Immunother 2009;58:15-23.

[13] Hudzik B, Szkodziński J, Lekston A, Gierlotka M, Poloński L, Gąsior M. Mean platelet volume-to-lymphocyte ratio: a novel marker of poor short-and long-term prognosis in patients with diabetes mellitus and acute myocardial infarction. J Diabetes Complications 2016;30:1097-102.

[14] Bansal R, Gupta HL, Goel A, Yadav M. Association of increased platelet volume in patients of chronic obstructive pulmonary disease: clinical implications. J Indian Acad Clin Med 2002;3:169-72.

[15] Ulasli SS, Ozyurek BA, Yilmaz EB, Ulubay G. Mean platelet volume as an inflammatory marker in acute exacerbation of chronic obstructive pulmonary disease. Pol Arch Med Wewn 2012;122:284-90.

[16] Agapakis D, Massa V, Hantzis I, Maraslis S, Alexiou E,
Imprialos $\mathrm{P}$, et al. The role of mean platelet volume in chronic obstructive pulmonary disease exacerbation. Respir Care 2016;61:44-9.

[17] Wang RT, Li JY, Cao ZG, Li Y. Mean platelet volume is decreased during an acute exacerbation of chronic obstructive pulmonary disease. Respirology 2013;18:1244-8.

[18] Vizioli L, Muscari S, Muscari A. The relationship of mean platelet volüme with the risk and prognosis of cardiovascular diseases. Int J Clin Pract 2009;63:1509-15.

[19] Milovanovic M, Nilsson E, Järemo P. Relationships between platelets and inflammatory markers in rheumatoid arthritis. Clin Chim Acta 2004;343:237-40.

[20] Kurtipek E, Bekci TT, Kesli R, Sami SS, Terzi Y. The role of neutrophil-lymphocyte ratio and platelet-lymphocyte ratio in exacerbation of chronic obstructive pulmonary disease. J Pak Med Assoc 2015;65:1283-7.

[21] Karadeniz G, Aktoğu S, Erer OF, Kır SB, Doruk S, Demir $\mathrm{M}$, et al. Predictive value of platelet-to-lymphocyte ratio in exacerbation of chronic obstructive pulmonary disease. Biomark Med 2016;10:701-10.

[22] Thomsen M, Ingebrigtsen TS, Marott JL, Dahl M, Lange P, Vestbo J, et al. Inflammatory biomarkers and exacerbations in chronic obstructive pulmonary disease. JAMA 2013;309:235361.

[23] Kocak MZ, Fidan K. Could the neutrophil-to-lymphocyte ratio be a marker of acute inflammation in chronic obstructive pulmonary disease? Eurasian J Med Invest 2018;2:8-11.

[24] Tamhane UU, Aneja S, Montgomery D, Rogers EK, Eagle KA, Gurm HS. Association between admission neutrophil to lymphocyte ratio and outcomes in patients with acute coronary syndrome. Am J Cardiol 2008;102:653-7.

[25] Fu H, Qin B, Hu Z, Ma N, Yang M, Wei T, et al. Neutrophiland platelet-to-lymphocyte ratios are correlated with disease activity in rheumatoid arthritis. Clin Lab 2015;61:269-73.

[26] Günay E, Sarınç Ulaşlı S, Akar O, Ahsen A, Günay S, Koyuncu T, et al. Neutrophil-tolymphocyte ratio in chronic obstructive pulmonary disease: a retrospective study. Inflammation 2014;37:374-80.

[27] Terradas R, Grau S, Blanch J, Riu M, Saballs P, Castells X. Eosinophil count and neutrophil-lymphocyte count ratio as prognostic markers in patients with bacteremia: a retrospective cohort study. PLoS One 2012; 7:e42860.

[28] Göçmen H, Çoban H, Yıldız A, Ursavaş A, Coşkun F, Ediger D. Is there any correlation between serum CRP level and haematological parameters with severity of disease in acute exacerbation of COPD? Respir Dis 2007;18:141-7.

[29] Gao P, Zhang J, He X, Hao Y, Wang K, Gibson PG. Sputum inflammatory cell-based classification of patients with acute exacerbation of chronic obstructive pulmonary disease. PLoS One 2013;31:e57678. 